\title{
Digital Micromirror Device (DMD)-based 3D printing of poly(propylene fumarate) scaffolds.
}

\author{
Eric J. Mott ${ }^{\mathrm{a}}$, Mallory Busso ${ }^{\mathrm{b}}$, Xinyi Luo ${ }^{\mathrm{b}}$, Courtney Dolder ${ }^{\mathrm{a}}$, Martha O. Wang ${ }^{\mathrm{c}}$, John P. Fisher ${ }^{\mathrm{c}}$, David \\ $\operatorname{Dean}^{\mathrm{a}, *}$ \\ a Department of Plastic Surgery, The Ohio State University, Columbus, OH 43210 \\ b Department of Neurological Surgery, Case Western Reserve University, Cleveland, OH 44106 \\ c Fischell Department of Bioengineering, University of Maryland, College Park, MD 20742
}

\begin{abstract}
Our recent investigations into the 3D printing of poly(propylene fumarate) (PPF), a linear polyester, using a DMD-based system brought us to a resin that used titanium dioxide $\left(\mathrm{TiO}_{2}\right)$ as an ultraviolet (UV) filter for controlling cure depth. However, this material hindered the 3D printing process due to undesirable lateral or "dark" curing (i.e., in areas not exposed to light from the DMD chip). Well known from its use in sunscreen, another UV filter, oxybenzone, has previously been used in conjunction with $\mathrm{TiO}_{2}$. In this study we hypothesize that combining these two UV filters will result in a synergistic effect that controls cure depth and avoids dark cure. A resin mixture (i.e., polymer, initiator, UV filters) was identified that worked well. The resin was then further characterized through mechanical testing, cure testing, and cytotoxicity testing to investigate its use as a material for bone tissue engineering scaffolds. Results show that the final resin eliminated dark cure as shown through image analysis. Mechanically the new scaffolds proved to be far weaker than those printed from previous resins, with compressive strength of $7.8 \pm 0.5 \mathrm{MPa}$ vs. $36.5 \pm$ 1.6 $\mathrm{MPa}$, respectively. The new scaffolds showed a $90 \%$ reduction in elastic modulus and a $74 \%$ increase in max strain. These properties may be useful in tissue engineering applications where resorption is required. Initial cytotoxicity evaluation was negative. As hypothesized, the use of $\mathrm{TiO}_{2}$ and oxybenzone showed synergistic effects in the 3D printing of PPF tissue engineering scaffolds.
\end{abstract}

Keywords: digital micromirror device, bone tissue engineering, 3D Printing, poly(propylene fumarate), resorbable, UV filter

\section{Introduction}

Solid freeform fabrication, or additive manufacturing, colloquially referred to as 3D printing, holds promise to enable patient specific $[1,2]$ tissue engineering resorbable implants (i.e., scaffolds) with controlled architecture because of the fidelity in which scaffold geometry can be designed and fabricated, thereby influencing important properties such as accuracy, repeatability, fit, mechanical performance, and degradability (resorption). For more than a decade our group has used photocrosslinking 3D printing

*Corresponding author

Email address: david.dean@osumc.edu (David Dean) 
devices to produce resorbable tissue engineering scaffolds. Initially, our efforts focused on stereolithography to 3D print poly(propylene fumarate) (PPF) for bone tissue engineering applications [3]. More recently, our effort has shifted to using a different photocrosslinking 3D printing technique that involves projecting a dynamic mask using Texas Instruments (Dallas, TX) digital light processing (DLP ${ }^{\circledR}$ ) technology, which utilizes a digital micromirror device (DMD) [4]. Many derivatives exist of DMD-based 3D printing systems with no recognizable standard to their nomenclature, yet all the systems use the same general principle: a DMD equipped projector shines a dynamic mask onto a vat of photopolymerizable resin in order to build a 3D part layer by layer. Therefore, the work herein is applicable to all of these devices.

In the literature, the nomenclature for these 3D printing systems has included continuous digital light processing (cDLP) [5], DLP-based 3D printing or stereolithography [6, 7], DMD projection printing (DMD-PP) [8], projection, mask projection, or DMD, micro-stereolithography $(\mu \mathrm{SL})[9,10,11]$, or dynamic mask stereolithography (DMS) [12]. These systems are usually top-down projection approaches, but some utilize bottom-up projection like our device. The key advantages of a bottom-up projection approach are quicker build times, higher resolution, and material conservation. However, a key disadvantage is material interaction (e.g., adhesion) with the resin containing tray, or "basement", which is usually solved through the use of specialized material coatings for these basements [13].

In pioneering the effort of using PPF in a bottom up projection approach, while successful, we found that a significant problem existed with the resin due to the UV-filter, $\mathrm{TiO}_{2}$. This is because $\mathrm{TiO}_{2}$ acts not only as a strong UV absorber, but also as a UV scatterer [18, 19]. This scattering of UV causes undesirable "dark cure" by activating the photoinitiator in regions not directly illuminated (i.e., areas intended to be "dark") leading to reduced efficiency and effectiveness of 3D printing [5]. Furthermore, these complications cause a loss of lateral resolution and accuracy [4]. Also, $\mathrm{TiO}_{2}$ is a pigment rather than a dye, as it does not dissolve and will fall out of solution over time. Other authors report similar complications when using ceramic particles $[14,15,16,17,6]$. These disadvantages suggest a solution: replacing $\mathrm{TiO}_{2}$ with a UV-filter that is a strong UV absorber, while causing little UV scattering, as well as being a dye (dissolving in the resin) rather than a pigment (suspending in the resin). The challenge in selecting such a material is biocompatibility and appropriate spectral absorption. Our preliminary work done to address this explored the UV-filter 2-Hydroxy-4-methoxybenzophenone (HMB), commercially known as oxybenzone. Our initial results (unpublished) addressed the fundamental parameter of photopolymer 3D printing, cure depth [29], or $C_{d}$, and showed that large amounts of HMB were required for the same $\mathrm{Cd}_{\mathrm{d}}$ as seen with $\mathrm{TiO}_{2}$, significantly impacting material properties. Further research however showed that there may be synergies in combining $\mathrm{TiO}_{2}$ and $\mathrm{HMB}$ as they have been commonly used together in sunscreen [20, 21, 22]. The aim of this work was to show that indeed these synergies occur, characterize them, and thereby correct dark cure, allowing for accurate and efficient 3D printing of PPF scaffolds for bone tissue engineering.

\section{Materials and Methods}

\subsection{Strategy for Finding Working Resins}

While all types of additives [23] should be considered in resin design, it was necessary for simplicity to start with as few as possible. Previous work in our lab focused on the use of a diluent, diethyl fumarate (DEF), a photoinitiator, bisacylphosphine oxide (BAPO), a pigment, $\mathrm{TiO}_{2}$, and recently, a dye, $\mathrm{HMB}$, as primary additives for our 3D printing resins [25]. Given the effects of these additives on the $\mathrm{Cd}$, our initial benchmark for 3D printing, a strategy was implemented toward finding a working resin, designated WR. This strategy is modeled in Figure 1.

\subsection{PPF Synthesis and Storage}

PPF was synthesized as described previously [24] and characterized using gel permeation 
chromatography to yield a number average molecular weight $\left(\mathrm{M}_{\mathrm{n}}\right)$ and polydispersity index (PDI). Based on previous experiments, the target $\mathrm{M}_{n}$ was $1200 \mathrm{Da}$. Later this PPF was heated to $\sim 50^{\circ} \mathrm{C}$ using a heat gun in order to decrease its viscosity and mixed with DEF (Sigma, St. Louis, MO) in a 3:1 ratio by mass to form a pre-resin. The 3:1 ratio proved useful for two reasons: (1) DEF serves as a diluent, thereby reducing the viscosity for easier mixing of resin components (i.e., photoinitiator, dye, and polymer), and (2) keeping the DEF at a level much lower than in a final resin (usually 1:1 ratio) allows for subsequent DEF to be used as a solvent for additives before being added to the 3:1 pre-resin. The 3:1 PPF to DEF mixture can be stored in a refrigerator $\left(4^{\circ} \mathrm{C}\right)$ until needed. The later addition of DEF to form a final resin additionally aids in keeping viscosity low enough for new material to flow in and re-wet the basement surface after each layer is formed during 3D printing and is further discussed in Section 2.7 [30, 31].

\subsection{Resin Formulation Definition}

Resin formulations were defined in relation to the combined mass of PPF and DEF, wherein additives were defined as weight percents of this mass. Unless otherwise specified, hereinafter all $\mathrm{w} / \mathrm{w} \%$ follow this convention. This was useful for updating resins to new formulations, thereby saving material, since the PFF to DEF ratio is usually held constant. Furthermore, this convention allows for a fast and intuitive understanding of the composition of a resin. Mathematically, Equation 1 gives the amount of each additive required for a resin given that the final mass of the resin has been specified:

$$
m_{a, i}=\left(\frac{M_{f}}{1+\sum_{i=1}^{n} c_{i}}\right) c_{i}
$$

where, $M_{f}$, is the desired final mass of a resin containing $n$ additives, and $c_{i}$, is the desired $\mathrm{w} / \mathrm{w} \%$ and $m_{a, i}$, is the mass to add of the $i^{\text {th }}$ additive. The part of the equation in the parentheses yields the required mass of combined PFF and DEF. Given the desired ratio of PPF and DEF, the relative mass of each additive is easily calculated.

\subsection{Resin Creation}

For the following resin preparation procedure, care should be taken to minimize resin exposure to any source of UV or near UV radiation. Upon preparation of a resin for $\mathrm{C}_{\mathrm{d}}$ testing, 3:1 PPF to DEF was allowed to warm to room temperature. DEF was measured out in a beaker such that when combined with 3:1 PPF to DEF, the solution ends up being the desired PPF to DEF ratio. The order of addition for additives to this DEF was determined through observation of solubility and optical impact. Additives more difficult to dissolve with little impact on increasing resin opacity were added first in order to visually assess solubility. For this study, BAPO (Ciba Specialty Chemicals, Tarrytown, NY) followed by HMB (Sigma, St. Louis, $\mathrm{MO})$ (if required) was measured out and dissolved in DEF under constant stirring and heat $\left(\sim 50^{\circ} \mathrm{C}\right)$. The solution was then allowed to cool to room temperature. 3:1 PPF to DEF was then directly measured out into this solution thereby preventing losses from transfer to another beaker. If the resin required rutile $\mathrm{TiO}_{2}$ (320 nm average particle size) (Sachtleben, Duisburg, Germany), this was measured out and added under constant stirring until well mixed. The addition of $\mathrm{TiO}_{2}$ was done last because it rapidly increases resin opacity and does not dissolve. 


\subsection{Cure Depth Tests}

To utilize a resin for 3D printing, a specification must be met for $\mathrm{C}_{\mathrm{d}}$. In this study, this specification was chosen to be $120-150 \mu \mathrm{m}$ based on our experience and manufacturer recommendation. This range of cure depths allows for $70-100 \mu \mathrm{m}$ of overcure, when printing at $50 \mu \mathrm{m} /$ layer, such that adequate layer-layer lamination occurs. To analyze this, it was necessary to perform cure depth tests (CDTs). A Perfactory 3 Mini Multi Lens (EnvisionTEC, Inc., Dearborn, MI) in UV and service mode was powered on and the bulb was allowed to warm for 20 minutes. A mask adjustment was performed on the Perfactory to calibrate for the desired light intensity using the built in radiometer over a water-white glass slide (Fisher, Waltham, MA) on the calibration plate. Care was taken to ensure that the slide used for this calibration was the same thickness as the slides to be used for CDTs, nominally $1 \mathrm{~mm}$. The "Curing Test" functionality was invoked on the Perfactory and the desired intensity was set. The built-in radiometer over a glass slide was used to measure the intensity immediately before a CDT was run. If the intensity was below the nominal or above plus $5 \mathrm{~mW} / \mathrm{dm}^{2}$ of it, the curing test functionality was reset until the measured intensity fell within this range. Upon this conformance, the built-in "Square" option for curing tests was selected and the desired exposure-time set. The slide used for the curing test calibration was then removed from the Perfactory. Using a $3 \mathrm{ml}$ transfer-pipet, six to seven drops of the resin being tested were placed in the middle of the slide. The slide was quickly aligned over the test square being projected by the Perfactory and the curing test was initiated. After the curing test was complete, the slide was removed turned upside down and dabbed off on an absorbent cloth so that all uncured material was removed. Using a Digimatic Series 500 digital caliper (Mitutoyo, Kawasaki, Japan), the cured test-sample and glass slide thickness was measured twice. From the average of these, the slide thickness was subsequently subtracted to find the thickness of the cured sample, or $\mathrm{C}_{\mathrm{d}}$. Inadvertent lateral curing caused by scattered light was noted if observed. The testsample was then carefully removed using a razor blade and qualitative observations of strength were noted such as whether it survived in one piece upon being removed and whether it could be subsequently handled.

\subsection{Scaffold Design}

A comparison scaffold was designed as previously described [25] to benchmark the resins. Figure 2 illustrates that the dimensions for the scaffold's inner base diameter, outer base diameter, base height, post diameter, post height, number of posts, overall height (including a base cap), support diameter, support height, and number of supports are $4.5 \mathrm{~mm}, 6 \mathrm{~mm}, 0.125 \mathrm{~mm}, 0.75 \mathrm{~mm}, 0.5 \mathrm{~mm}, 21,10.125 \mathrm{~mm}, 0.375$ $\mathrm{mm}, 1 \mathrm{~mm}$, and 46 , respectively.

\subsection{DMD-based 3D Printing}

The Perfactory in UV mode was powered on and the bulb was allowed to warm for 20 minutes. Following this, a 48 -field mask adjustment was performed at $350 \mathrm{~mW} / \mathrm{dm}^{2}$, the light intensity used for 3D printing. A build of twelve scaffolds of WR was done using a modified M-type basement (EnvisionTEC, Inc., Dearborn, MI) that utilized a 112.7 x $150.8 \mathrm{~mm}$ inner containment ring (Figure 3) to reduce the required material to approximately $35 \mathrm{ml}$. The full build-style implemented is outlined in Table 1 and these parameters are depicted in Figure 4. These build-style settings are critical in the successful outcome of a print. The burn-in range and standard range allow for two different sets of these settings, the latter is required and used for most of the build, the prior is usually set to a higher exposure to allow for adequate attachment to the build platform of initial layers. These settings are important due to basement adhesion and resin flow-back into the void left by the previous built layer. Adhesive force of a crosslinked polymer in contact with a surface (in our case, the basement) increases with separation velocity, which is the rate the polymer is removed from the surface, and contact time, which is the duration the polymer is in contact with 
the surface [28]. For this reason, we chose relatively slow separation velocities and no wait time. A large separation distance was chosen to completely remove the part from the resin after each layer, which better allows for resin flow-back, especially when using viscous resins. Finally, axis-positioning rate was kept low to avoid trapping air and part damage upon reentrance into the resin. Likewise, tilt-positioning rate was kept low to avoid resin sloshing. A build of the same scaffold design using previously described methods [4] was done for comparison using a benchmark resin, BR, which contained 1:1 PPF to DEF, 1\% BAPO, and $3 \% \mathrm{TiO}_{2}$.

\begin{tabular}{|c|c|c|c|c|c|c|}
\hline & & \multicolumn{2}{|c|}{ Separation } & \multirow{2}{*}{$\frac{\text { Positioning }}{\text { Velocity }[\mu \mathrm{m} / \mathrm{s}]}$} & \multicolumn{2}{|c|}{ Waiting Time Before } \\
\hline & & Distance $[\mu \mathrm{m}]$ & Velocity $[\mu \mathrm{m} / \mathrm{s}]$ & & Separation [s] & Positioning [s] \\
\hline $\begin{array}{l}\text { Burn-In Range } \\
(120 / 70 / 1000)\end{array}$ & $\begin{array}{r}\text { Axis } \\
\text { Tilt }\end{array}$ & $\begin{array}{l}4000 \\
7000\end{array}$ & $\begin{array}{c}2000 \\
800\end{array}$ & $\begin{array}{r}1000 \\
800\end{array}$ & $\begin{array}{l}0 \\
0\end{array}$ & $\begin{array}{l}0 \\
2\end{array}$ \\
\hline $\begin{array}{l}\text { Standard Range } \\
(60 / 4 / \text { n.a. })\end{array}$ & $\begin{array}{r}\text { Axis } \\
\text { Tilt }\end{array}$ & $\begin{array}{l}4000 \\
4500\end{array}$ & $\begin{array}{l}3000 \\
1050\end{array}$ & $\begin{array}{r}300 \\
1050\end{array}$ & $\begin{array}{r}0 \\
0.5\end{array}$ & $\begin{array}{l}0 \\
0\end{array}$ \\
\hline
\end{tabular}

Exposure Time [s], Waiting Time Before Exposure [s], and Thickness $[\mu \mathrm{m}]$ in parentheses.

Table 1: Perfactory build-style settings. Additionally, the enhanced resolution module (ERM) was enabled, which uses anti-aliasing to improve accuracy. The baseplate option, which 3D prints a base layer of material, was disabled.

\subsection{Processing of Scaffolds}

Finished scaffolds were processed by repeated rinses of acetone and $70 \% \mathrm{v} / \mathrm{v}$ ethanol for 15 seconds using wash bottles. Immediately following this, the specimens were rinsed for five-seconds with distilled water and blown clean and dry using compressed air. Afterwards, the scaffolds were removed from the build platform using a razor blade and subsequently post-cured in a ProCure 350 UV chamber (3DSystems, Rock Hill, SC) for $480 \mathrm{~min}$ in order to increase strength. Scaffolds were stored at room temperature in the dark.

\subsection{Compression Tests}

Scaffolds built with WR and BR were tested in compression to failure at a strain rate of $0.1 \mathrm{~mm} / \mathrm{s}$ using an MTS Landmark (Eden Prairie, MN) outfitted with a Model $41500 \mathrm{lb}_{\mathrm{f}}$ load cell (Honeywell, Morristown, NJ) and basic upper/lower platens. Prior to testing, the initial length of each scaffold was measured with a Series 293-185 QuantuMike digital micrometer (Mitutoyo, Kawasaki, Japan). Elastic modulus was calculated by taking the slope of the initial linear part of the stress-strain curve. Compressive strength was taken to be the maximum compressive stress during the test. To determine stress, the average cross sectional area of the top end (end built last during 3D printing) of all the scaffolds of a resin type was used. Strain was derived via displacement data from the MTS Landmark and initial lengths of the scaffolds. Data reported is mean \pm standard deviation.

\subsection{PFF to DEF Ratio Effect on $C_{d}$}

To understand the effect of varying the PPF to DEF ratio of a resin on the $\mathrm{C}_{\mathrm{d}}$ for a given exposure, CDTs were performed as described in the previous section at an intensity of $350 \mathrm{~mW} / \mathrm{dm}^{2}$ and an exposure time of $60 \mathrm{~s}$ using WR prepared as previously described. PPF to DEF ratios of 1.5:1 through 0.7:1 were tested in 0.2:1 increments by increasing the DEF content of the resin, while resolving the additives back to their original concentrations using Equation 2 as discussed in the following section. 


\begin{tabular}{|c|c|c|c|c|c|c|c|c|c|c|c|c|}
\hline Resin I.D & \multicolumn{3}{|c|}{ Initial Formulation } & \multicolumn{3}{|c|}{ Update 1} & \multicolumn{3}{|c|}{ Update 2} & \multicolumn{3}{|c|}{ Update 3} \\
\hline 1 & 1 & 0 & 0 & 1 & 0 & 1 & 1 & 0 & 2 & 1 & 0 & 3 \\
\hline 3 & 1 & 20 & 0 & 1 & 20 & 1 & 1 & 20 & 2 & 1 & 20 & 3 \\
\hline 4 & 1 & 30 & 0 & 1 & 30 & 1 & 1 & 30 & 2 & 1 & 30 & 3 \\
\hline 5 & 2 & 0 & 0 & 2 & 0 & 1 & 2 & 0 & 2 & 2 & 0 & 3 \\
\hline 8 & 2 & 30 & 0 & 2 & 30 & 1 & 2 & 30 & 2 & 2 & 30 & 3 \\
\hline 9 & 3 & 0 & 0 & 3 & 0 & 1 & 3 & 0 & 2 & 3 & 0 & 3 \\
\hline 10 & 3 & 10 & 0 & 3 & 10 & 1 & 3 & 10 & 2 & 3 & 10 & 3 \\
\hline 11 & 3 & 20 & 0 & 3 & 20 & 1 & 3 & 20 & 2 & 3 & 20 & 3 \\
\hline 12 & 3 & 30 & 0 & 3 & 30 & 1 & 3 & 30 & 2 & 3 & 30 & 3 \\
\hline
\end{tabular}

Table 2: Update schedules for formulations used for determining the cure depth design space for resins using BAPO, HMB, and $\mathrm{TiO}_{2}$. Each resin was updated three times by increasing $\mathrm{TiO}_{2}$. Values are $\mathrm{m} / \mathrm{m} \%$ as described in section 2.3 .

\subsection{Characterizing $C_{d}$ Design Space}

To determine the $\mathrm{C}_{\mathrm{d}}$ design space, twelve initial resins were created in $10 \mathrm{~g}$ batches each as described in previous sections using PPF with $\mathrm{Mn}_{\mathrm{n}} 1300 \mathrm{~g} / \mathrm{mol}$ and (PDI = 1.74). These resins were updated according to the schedule outlined in Table $2 . \mathrm{TiO}_{2}$ was chosen as the additive to update for the mixture as it does not dissolve and makes the resin opaque. The updating process for a resin involves weighing the remaining resin, calculating the amount of $\mathrm{TiO}_{2}$ to add, and mixing in the $\mathrm{TiO}_{2}$ under stirring until it is well mixed. Equation 2 represents the general mathematical expression for calculating how much of each resin component to add when updating a resin:

$$
m_{a, i}=\left(\frac{M_{r}}{1+\sum_{i=1}^{n} c_{i}}\right)\left(\begin{array}{ll}
c_{i}^{\prime} & c_{i}
\end{array}\right)
$$

where, $M_{r}$, is the mass of remaining resin of $n$ additives, and $c_{i}$, is the current $\mathrm{w} / \mathrm{w} \%, c_{i}^{\prime}$, is the desired $\mathrm{w} / \mathrm{w} \%$, and $m_{a, i}$, is the additional mass to add of the $i^{\text {th }}$ additive (in this case, $\mathrm{TiO}_{2}$ ). CDTs were done as previously described at an intensity of $260 \mathrm{~mW} / \mathrm{dm}^{2}$; however an exposure time of $120 \mathrm{~s}$ was used in these experiments. This lengthy exposure, beyond the time we prefer, was necessitated by a manufacturerrecommended upgrade to the Perfactory, which placed a UV shortpass filter (i.e., allows only UV light through, in this machine it is cutting out all visible light wavelengths) on the lens in order to remove longer wavelengths. The result was a much lower attainable intensity, which required increasing the exposure time. As noted, increasing the exposure time risks increasing adhesive forces with the basement, which in turn can lead to low yield or complete build failure. This filter was eventually removed. Even though the data acquired from this experiment does not directly correspond with ideal 3D printing conditions and the conditions used elsewhere in this study, the shape of the resulting data provides important insight into potential resin chemistries that will result in successful 3D printing.

\subsection{Characterizing Dark Cure}

CDTs of BR and WR were produced as previously described at $350 \mathrm{~mW} / \mathrm{dm}^{2}$ over six exposure times 
from $60 \mathrm{~s}$ to $160 \mathrm{~s}$. The CDTs were imaged for dark cure analysis using a Model A1549 back-camera (Apple, Cupertino, CA) held parallel to the CDT sample at a distance of $10 \mathrm{~cm}$ using a ring stand. The sample was held via a custom jig $12.5 \mathrm{~cm}$ off of a black velveteen (Robert Kaufman Fabrics, Los Angeles, CA) backdrop. A lamp with a diffuser was used to evenly light the sample positioned to eliminate shadows. These steps were taken to create an even black background for images, which were subsequently analyzed in ImageJ (http://imagej.nih.gov/ij/) through the following procedure as illustrated in Figure 5. The entire sequence of images for a resin was imported into ImageJ and the following image processing was conducted. First, the images were cropped just larger than the largest dark cure region. The image stack was then converted to 8-bit grayscale. The contrast was then set to maximum in order to partition the cured area and the image stack was then converted to binary via the Make Binary tool. Following this, the Remove Outliers tool, with threshold set to 100 and radius to 20 pixels, was run to remove bright islands not part of the main cured body. Using the Threshold adjustment, the stack was reconverted to binary in order to invert the black and white regions. The Remove Outliers process was run again with prior settings to fill any holes in the main cured body. Subsequently, the image stack was repeatedly put through the Despeckle function to remove any remaining islands. Finally, the binary Close tool was run to remove edge defects. Using the Analyze Particles tool, the total cured area was calculated including the dark cure region and the sample. Normalized non-dimensional dark cure areas were then determined by subtracting out, and dividing by, the exposed area.

\subsection{Cytotoxicity}

Cytotoxicity was evaluated as previously described [24]. Briefly, thin films were prepared by spreading a thin layer of WR over a water white glass slide, compressing the resin evenly using a cover slide, and curing for $30 \mathrm{~min}$ in a UV chamber. Subsequently, the films were scored in approximately $1 \mathrm{x} 1 \mathrm{~cm}$ squares using a razor blade to aid in final separation and then cured for $450 \mathrm{~min}$ in a UV chamber with the cover slide replaced. Finally, the fully cured square films were separated. Under sterile conditions, the thin films were soaked for $15 \mathrm{~min}$ in Dulbecco's phosphate buffered saline solution (DBPS) (Life Technologies, Carlsbad, CA) followed by $70 \% \mathrm{v} / \mathrm{v}$ acetone for 30, 20, and 10 minutes, separated by soaks in fresh DPBS for 15 min. Next, the thin films were soaked for 15 min twice and then 4 hours in fresh DPBS before being placed in FBS overnight. Next, with media removed, these serum-soaked thin films were placed directly on an L929 cell (Sigma-Aldrich, St. Louis, MO) monolayer, pre-cultured to 80\% confluence in a 24-well plate (Corning Life Sciences, Corning, NY). $150 \mu 1$ of media comprised of Minimum Essential Medium (MEM) (Sigma-Aldrich, St. Louis, MO), 10\% v/v horse serum (SigmaAldrich, St. Louis, MO), and 1\% v/v penicillin/streptomycin solution (Life Technologies, Carlsbad, CA) was then added to each well. A solution of $2 \mu \mathrm{M}$ calcein AM and $4 \mu \mathrm{M}$ ethidium homodimer-1 (EthD-1) was prepared in DPBS using an L-3224 Live/Dead kit (Life Technologies, Carlsbad, CA). After 24 hours of cell culture with the thin films, the media was removed, and $150 \mu \mathrm{l}$ of live/dead solution was added to each well. This was left to soak for $30 \mathrm{~min}$ at room temperature in the dark, after which, the remaining solution was removed and replaced by $20 \mu 1$ of PBS. Images of the L929 monolayer were captured at 10x on an Olympus (Center Valley, PA) CKX41 fluorescence microscope with an Olympus DP72 12.8 MP digital camera.

\section{Results}

\subsection{Identifying a Working Resin}

The implemented resin exploration strategy resulted in over three hundred CDTs performed on over forty different resin chemistries, four intensities, and twelve exposure times to identify a resin that met the $120-130 \mu \mathrm{m}$ cure depth, eliminated dark cure at this specification, and successfully 3D printed an accurate scaffold. This working resin, WR, is composed of 1:1 PPF to DEF, $1 \% \mathrm{BAPO}, 1 \% \mathrm{TiO}_{2}$, and $28 \% \mathrm{HMB}$, tested at $350 \mathrm{~mW} / \mathrm{dm}^{2}$ for $60 \mathrm{~s}$. The high concentration of HMB proved necessary to offset the dark cure 
caused by $\mathrm{TiO}_{2}$.

\subsection{Scaffolds}

A successful build was done at 60 s/layer exposure instead of 150 s/layer using WR without manual intervention [5] to compensate for dark cure, which reduced the build time nearly $75 \%$. Figure 7 shows the apparent increase in accuracy of a benchmark scaffold printed with WR over the same scaffold printed with BR. In depth quantitative analysis of this is possible and has been done previously in this lab [4]; however, an accuracy analysis was deemed beyond the scope of this study. In examining Figure 7, the scaffold built with WR clearly eliminated "dark cure" artifacts as can be seen in the improved surface smoothness as compared to the BR scaffold, which has many particulates on the surface. Noticeable scaffold shrinkage was observed for both resins. Their inner and outer diameters were measured at the end opposite of the supports. Average \pm standard error shrinkage for outer and inner diameters was $6.7 \pm 0.8 \%$ and $6.9 \pm 0.8 \%$, respectively for BR $(n=9)$, and $10.4 \pm 0.6 \%$ and $12.6 \pm 0.7 \%$, respectively for WR $(n=4)$.

\subsection{Mechanical Testing}

The average stress-strain curve is shown in Figure 8 for scaffolds built with WR and BR tested in compression. Five scaffolds built with BR were compressed to failure yielding an average elastic modulus (taken as the slope of the initial linear region) of $837.2 \pm 18.9 \mathrm{MPa}$ and an average compressive strength of $36.5 \pm 1.6 \mathrm{MPa}$. Four WR scaffolds were compressed to failure and had an average elastic modulus of 85.0 $\pm 1.0 \mathrm{MPa}$ and an average compressive strength of $7.8 \pm 0.5 \mathrm{MPa}$. The failure mode for the scaffolds tested of both resins appeared to be buckling, however, scaffolds built with BR experienced sudden catastrophic failure indicative of being brittle, while WR experienced a more ductile failure.

\subsection{Effect of PPF to DEF ratio on $C_{d}$}

Five different PPF to DEF ratios for WR were evaluated as shown in Figure 9 and showed no significant effect on the $\mathrm{C}_{\mathrm{d}}$. As expected, we qualitatively observed a noticeable decrease in resin viscosity as DEF was increased due to DEF having a much lower viscosity than PPF.

\subsection{Dark Cure}

Results of varying the exposure time on BR and WR are plotted in Figure 10. As exposure time was increased, the dark cure area increased as well, but was greatly minimized in the case of WR.

\section{6. $\mathrm{C}_{d}$ Design space using $\mathrm{HMB}, \mathrm{TiO}_{2}$, and $\mathrm{BAPO}$}

The results of the CDTs varying BAPO, oxybenzone, and $\mathrm{TiO}_{2}$ are shown in Figure 11. An overall decrease in $\mathrm{C}_{\mathrm{d}}$ occurs for increasing $\mathrm{BAPO}, \mathrm{TiO}_{2}$, and $\mathrm{HMB}$. As expected, dark cure occurs where $\mathrm{TiO}_{2}$ is present and HMB is low or absent. Furthermore, an increase in BAPO level greatly increases the likelihood of dark cure presence. In Figure 11, filled-in markers represent trials with dark cure occurrence and the target range $(120-150 \mu \mathrm{m})$ for $\mathrm{C}_{\mathrm{d}}$ is shown in grey.

\subsection{Cytotoxicity}

Cells were cultured in direct contact with serum-soaked WR thin films. Figure 12 shows a qualitatively negative (i.e., non-cytotoxic) result when compared to the positive and negative controls. Cell morphology was normal compared to the positive control. The bright area is caused by reflected light from the thin film.

\section{Discussion}

The resin exploration strategy presented here was successful in discovering a working resin, WR, which contained 1:1 PPF to DEF, $1 \% \mathrm{BAPO}, 1 \% \mathrm{TiO}_{2}$, and $28 \% \mathrm{HMB}$. The synergistic effects of combining $\mathrm{TiO}_{2}$ 
and HMB allowed for automated (i.e., no need for manual intervention during 3D printing due to dark cure) and high yield 3D printing of scaffolds. This resin, while utilizing a large amount of HMB, greatly reduced the amount of $\mathrm{TiO}_{2}$. CDTs clearly showed that this chemistry eliminated dark cure, wherein the $\mathrm{HMB}$ did most of the dual work of reducing $\mathrm{C}_{\mathrm{d}}$ and absorbing the scattered light of $\mathrm{TiO}_{2}$. While $\mathrm{TiO}_{2}$ did contribute to reducing $\mathrm{C}_{\mathrm{d}}$, there was an observable increase in strength of the CDTs as well. As expected, these synergies allowed for the successful printing of a modular type benchmark scaffold (Figure 7) without the drawbacks originally reported with our early use of $\mathrm{TiO}_{2}$ in a PPF 3D printing process [5]. Furthermore, the green strength [29], or the strength of the scaffolds immediately out of the 3D printer, was high enough to undergo the harsh processing conditions necessary to obtain the high accuracy and cleanliness desired.

Noticeable shrinkage did occur as expected, measuring slightly larger with WR than BR. A high degree of shrinkage can be a disadvantage in that only so much can be done in the design of a scaffold to compensate for it. While shrinkage can be addressed in software, it is usually limited to an isotropic scaling factor for correction, which often falls short, as shrinkage is an anisotropic problem [4]. Therefore, one goal of resin development should be to reduce shrinkage as much as possible. This can be achieved readily through the incorporation of solid fillers that do not react with the actinic light, wherein the shrinkage will be directly proportional to the concentration of such fillers [33]. This is likely the reason why BR displayed less shrinkage than WR due to its greater $\mathrm{TiO}_{2}$ content. While a zero-shrinkage resin may seem desirous, it is also important to consider in vivo swelling, which will tend to enlarge scaffold geometry, although anisotropically [34].

Mechanical testing in compression showed a large difference in performance between WR and BR. The high concentration of $\mathrm{HMB}$ coupled with the reduction of $\mathrm{TiO}_{2}$ in $\mathrm{WR}$ caused the mechanical strength to be greatly reduced. However, the elasticity of WR is considerably higher than BR, which depending on the application could be a strong advantage. For example, it may improve resorption due to lower crosslink density [32]. A lower elastic modulus of a photocrosslinked polymer is usually an indication of a lower crosslink density [28], which is reasonable to infer in this case due to the large concentration of HMB competing with the initiator for UV absorption thereby hindering crosslink conversion.

In varying the PPF to DEF ratio on the $C_{d}$ of WR, no significant change occurred over the evaluated range. However, while this indicates the depth of penetration of UV effectively remained unchanged, the extent of crosslinking taking place at the higher concentrations of DEF is reduced, which will ultimately reduce mechanical strength [27]. However, reducing the viscosity is useful for clearing out internal pores of uncured resin, which ultimately leads to higher accuracy throughout a scaffold, suggesting further investigation into the use of lower PPF to DEF ratios or heating of the resin during printing.

As expected, lateral loss in resolution caused by light scattering, or dark cure, was observed to correlate with exposure time (Figure 10). The logarithmic response of the area is similar to that of $C_{d}$ and may be modeled as such [26]. Other investigators have applied Mie Theory for design purposes to compensate for the scattering-induced loss of accuracy [16]. However, in cases such as ours, where high ceramic loading is unnecessary, the elimination of dark cure is desirable. Figure 6 clearly shows that under the conditions of 3D printing, the use of WR achieves this due to the incorporation of a pure UV absorber, HMB. However, due to the presence of $\mathrm{TiO}_{2}$, dark cure will always occur if enough energy is put into the resin. Figure 10 demonstrates this showing the monotonic increase in dark cure area with increasing exposure. This suggests that minimizing exposure time will improve accuracy for a given light intensity. This of course is limited by the reduction in mechanical strength and $\mathrm{C}_{\mathrm{d}}$. Lower exposure time is also beneficial in reducing the likelihood of strong adhesive forces between the basement and the last cured layer as discussed earlier, which in part, increases the likelihood of a successful build. Regardless, optimization of exposure time should be done as it also leads to significant reduction in build time.

A reasonable design space was characterized by varying $\mathrm{BAPO}, \mathrm{TiO}_{2}$, and $\mathrm{HMB}$ as a means to form insight on optimizing these resin components as shown in Figure 11. A plausible range of useful resin chemistries for a given exposure was determined. This range was quite limited due to dark cure formation and tight specification for $\mathrm{C}_{\mathrm{d}}$. However, it suggests going to $3 \%$ BAPO because the target $\mathrm{C}_{\mathrm{d}}$ can be achieved without the use of $\mathrm{TiO}_{2}$, thereby eliminating the chance of dark cure. However the drawback in doing so may be sufficient strength as it requires a high concentration of HMB. Even so, at this initiator 
level, $\mathrm{TiO}_{2}$ could be kept at $1 \%$, allowing $\mathrm{HMB}$ to be reduced to $20 \%$, which should increase mechanical strength.

From the design of the resin, to the manner in which it is printed, and to the way it is post-processed, resolution is impacted at every step in the process. It is important to start with the best possible resin, thereby increasing the likelihood that subsequent steps will need less optimization and result in little impact on final scaffold accuracy. The accuracy of the scaffold both internally and externally is of the utmost importance as it effects not only how the scaffold will interact with the environment it is placed in, but how cells and tissue will respond when incorporated in it. For example, scaffold geometry directly impacts degradation, wall shear stress, and nutrient transport in perfusion, which will have great effect on cell adhesion, proliferation, and differentiation both in the body and bioreactors. Control of this can only be done through a well-tuned system that focuses on the material and its fabrication, in this case, DMD-based 3D printing of PPF.

\section{Conclusion}

A resin exploration strategy was successfully implemented and an effective resin, WR, was discovered that allowed for efficient DMD-based 3D printing of PPF scaffolds without manual intervention as previously reported [5]. These scaffolds were mechanically tested in compression, wherein a high degree of elasticity was observed, which to the knowledge of these authors has not been reported before for PPF scaffolds. PFF to DEF ratio was varied for WR and found to have no significant impact on $\mathrm{C}_{\mathrm{d}}$. Dark cure was characterized for WR, showing a significant reduction over BR, our previous resin. This suggests that it would be useful to explore stronger, biocompatible, pure UV absorbers to combat the light scattering of ceramics such as $\mathrm{TiO}_{2}$. In examining the potential design space for the initiator (BAPO), dye (HMB), and pigment $\left(\mathrm{TiO}_{2}\right)$ used, it was found that increasing the initiator to a $3 \%$ level will likely show improvement over WR. Furthermore, due to a high level of HMB required to achieve desired 3D printing outcomes, cytotoxicity was explored. Live/dead assays of thin films resulted in negative cytotoxicity. While out of scope for this study, other authors report degradation products will likely be non-cytotoxic as well [35]. All results combined validated the expectation of synergistic effects when combining $\mathrm{TiO}_{2}$ and $\mathrm{HMB}$ as UVfilters for resolution control in the DMD-based 3D printing of PPF scaffolds for bone tissue engineering.

\section{Acknowledgements}

We wish to acknowledge useful discussions with Jonathan Wallace and two of the authors (EM and DD) while they were at Case Western Reserve University prior to Fall 2013. This project received partial support from NIH grant R01-AR061460, and the Army, Navy, NIH, Air Force, VA, and Health Affairs to support the AFIRM II effort under award No. W81XWH-14-2-0004. The US Army Medical Research Acquisition Activity is the awarding and administering acquisition office for award No. W81XWH-14-2-0004. Opinions, interpretations, conclusions, and recommendations are those of the authors and are not necessarily endorsed by the US Department of Defense.

\section{References}

[1] R. A. Levy, S. Guduri, R. H. Crawford, Preliminary experience with selective laser sintering models of the human temporal bone, AJNR Am J Neuroradiol 15 (3) (1994) 473-7.

[2] F. W. Zonneveld, S. Lobregt, J. C. van der Meulen, J. M. Vaandrager, Three-dimensional imaging in craniofacial surgery, World J Surg 13 (4) (1989) 328-42.

[3] M. N. Cooke, J. P. Fisher, D. Dean, C. Rimnac, A. G. Mikos, Use of stereolithography to manufacture critical-sized 3d biodegradable scaffolds for bone ingrowth, J Biomed Mater Res B Appl Biomater 64 (2) (2003) 65-9. doi:10.1002/jbm.b.10485. 
[4] J. Wallace, M. O. Wang, P. Thompson, M. Busso, V. Belle, N. Mammoser, K. Kim, J. P. Fisher, A. Siblani, Y. Xu, J. F. Welter, D. P. Lennon, J. Sun, A. I. Caplan, D. Dean, Validating continuous digital light processing (cDLP) additive manufacturing accuracy and tissue engineering utility of a dye- initiator package, Biofabrication 6 (1) (2014) 015003. doi:10.1088/1758-5082/6/1/015003.

[5] D. Dean, W. Jonathan, A. Siblani, M. O. Wang, K. Kim, A. G. Mikos, J. P. Fisher, Continuous digital light processing (cDLP): Highly accurate additive manufacturing of tissue engineered bone scaffolds, Virtual Phys Prototyp 7 (1) (2012) 13-24. doi:10.1080/17452759.2012.673152.

[6] G. Mitteramskogler, R. Gmeiner, R. Felzmann, S. Gruber, C. Hofstetter, J. Stampfl, J. Ebert, W. Wachter, J. Laubersheimer, Light curing strategies for lithography-based additive manufacturing of customized ceramics, Additive Manufacturing 1-4 (0) (2014) 110 - 118, inaugural Issue. doi:http://dx.doi.org/10.1016/j.addma.2014.08.003.

URL

http://www.sciencedirect.com/science/article/pii/s2214860414000086

[7] M. Hatzenbichler, M. Geppert, R. Seemann, J. Stampfl, Additive manufacturing of photopolymers using the texas instruments dlp lightcrafter (2013). doi:10.1117/12.2001651.

URL http://dx.doi.org/10.1117/12.2001651

[8] L.-H. Han, S. Suri, C. E. Schmidt, S. Chen, Fabrication of three-dimensional scaffolds for heterogeneous tissue engineering, Biomed Microdevices 12 (4) (2010) 721-5. doi:10.1007/s10544-010-94252.

[9] C. Sun, N. Fang, D. Wu, X. Zhang, Projection micro-stereolithography using digital micromirror dynamic mask, Sensors and Actuators A: Physical 121 (1) (2005) 113 - 120. doi:http://dx.doi.org/10.1016/j.sna.2004.12.011.

URL

http://www.sciencedirect.com/science/article/pii/s0924424704008672

[10] V. Varadan, X. Jiang, V. Varadan, Microstereolithography and other fabrication techniques for 3D MEMS, Wiley, 2001.

URL http://books.google.com/books?id=bStTAAAAMAAJ

[11] Y. Lu, G. Mapili, G. Suhali, S. Chen, K. Roy, A digital micro-mirror device-based system for the microfabrication of complex, spatially patterned tissue engineering scaffolds, J Biomed Mater Res A 77 (2) (2006) 396-405. doi:10.1002/jbm.a.30601.

[12] J. Stampfl, H. Pettermann, R. Liska, Bioinspired cellular structures: Additive manufacturing and mechanical properties, in: P. Gruber, D. Bruckner, C. Hellmich, H.-B. Schmiedmayer, H. Stachelberger, I. C. Gebeshuber (Eds.), Biomimetics-Materials, Structures and Processes, Springer, 2011, Ch. 6, pp. 105-123. doi:10.1007/978-3-642-11934-7.

[13] Y. Pan, C. Zhou, Y. Chen, A fast mask projection stereolithography process for fabricating digital models in minutes, Journal of Manufacturing Science and Engineering 134 (5) (2012) 051011051011. URL http://dx.doi.org/10.1115/1.4007465

[14] C. Hinczewski, S. Corbel, T. Chartier, Ceramic suspensions suitable for stereolithography, Journal of the European Ceramic Society 18 (6) (1998) 583 - 590. doi:http://dx.doi.org/10.1016/S09552219(97)00186-6.

URL

http://www.sciencedirect.com/science/article/pii/s0955221997001866 
[15] C. Provin, S. Monneret, Complex ceramic-polymer composite microparts made by microstereolithography, Electronics Packaging Manufacturing, IEEE Transactions on 25 (1) (2002) 59-63. doi:10.1109/TEPM.2002.1000484.

[16] C. Sun, X. Zhang, Experimental and numerical investigations on microstereolithography of ceramics, Journal of Applied Physics 92 (8) (2002) 4796-4802. doi:http://dx.doi.org/10.1063/1.1503410.

URL

http://scitation.aip.org/content/aip/journal/jap/92/8/10.1063/1.1503 410

[17] W. Chen, S. Kirihara, Y. Miyamoto, Fabrication of three-dimensional micro photonic crystals of resinincorporating tio2 particles and their terahertz wave properties, Journal of the American Ceramic Society 90 (1) (2007) 92-96. doi:10.1111/j.1551-2916.2006.01377.x.

URL http://dx.doi.org/10.1111/j.1551-2916.2006.01377.x

[18] H. Yang, S. Zhu, N. Pan, Studying the mechanisms of titanium dioxide as ultraviolet-blocking additive for films and fabrics by an improved scheme, Journal of Applied Polymer Science 92 (5) (2004) 32013210. doi:10.1002/app.20327.

URL http://dx.doi.org/10.1002/app.20327

[19] A. P. Popov, A. V. Priezzhev, J. Lademann, R. Myllylä, Tio 2 nanoparticles as an effective UV-B radiation skin-protective compound in sunscreens, Journal of Physics D: Applied Physics 38 (15) (2005) 2564. URL http://stacks.iop.org/0022-3727/38/i=15/a=006

[20] R. Stokes, B. Diffey, The feasibility of using fluorescence spectroscopy as a rapid, non-invasive method for evaluating sunscreen performance, Journal of Photochemistry and Photobiology B: Biology 50 (2-

3) (1999) 137 - 143. doi:http://dx.doi.org/10.1016/S1011-1344(99)00084-6.

URL

http://www.sciencedirect.com/science/article/pii/s1011134499000846

[21] N. Serpone, A. Salinaro, A. V. Emeline, S. Horikoshi, H. Hidaka, J. Zhao, An in vitro system- atic spectroscopic examination of the photostabilities of a random set of commercial sunscreen lotions and their chemical UVB/UVA active agents, Photochem. Photobiol. Sci. 1 (2002) 970-981. doi:10.1039/B206338G.

URL http://dx.doi.org/10.1039/B206338G

[22] J. Lademann, S. Schanzer, U. Jacobi, H. Schaefer, F. Pflücker, H. Driller, J. Beck, M. Meinke, A. Roggan, W. Sterry, Synergy effects between organic and inorganic UV filters in sunscreens, J Biomed Opt 10 (1) (2005) 14008. doi:10.1117/1.1854112.

[23] G. Pritchard, Plastics Additives: An A-Z Reference, Polymer Science and Technology Series, Springer Netherlands, 1998.

URL http://books.google.com/books?id=Oa7d1BhGAJMC

[24] M. O. Wang, J. M. Etheridge, J. A. Thompson, C. E. Vorwald, D. Dean, J. P. Fisher, Evaluation of the in vitro cytotoxicity of cross-linked biomaterials, Biomacromolecules 14 (5) (2013) 1321-9. doi: $10.1021 / \mathrm{bm} 301962 \mathrm{f}$.

[25] M. O. Wang, C. E. Vorwald, M. L. Dreher, E. J. Mott, M.-H. Cheng, A. Cinar, H. Mehdizadeh, S. 
Somo, D. Dean, E. M. Brey, J. P. Fisher, Evaluating 3d-printed biomaterials as scaffolds for vascularized bone tissue engineering, Adv Mater doi:10.1002/adma.201403943.

[26] S. P. Gentry, J. W. Halloran, Depth and width of cured lines in photopolymerizable ceramic suspensions, Journal of the European Ceramic Society 33 (10) (2013) 1981 - 1988. doi:http://dx.doi.org/10.1016/j.jeurceramsoc.2013.02.033.

URL

http://www.sciencedirect.com/science/article/pii/s0955221913001386

[27] J. P. Fisher, D. Dean, A. G. Mikos, Photocrosslinking characteristics and mechanical properties of diethyl fumarate/poly(propylene fumarate) biomaterials, Biomaterials 23 (22) (2002) 4333-43. doi:10.1016/S0142-9612(02)00178-3.

[28] A. M. Grillet, L. M. Gloe, N. B. Wyatt, Polymer gel rheology and adhesion, INTECH Open Access Publisher, 2012. doi:10.5772/36975. URL http://www.intechopen.com/books/rheology/rheologyand-adhesion-of-polymer-gels.

[29] P. F. Jacobs, Rapid prototyping \& manufacturing: fundamentals of stereolithography, Society of Manufacturing Engineers, 1992.

[30] J. Gaspar, P.J. Bartolo, D.J. Duarte, Cure and rheological analysis of reinforced resins for stereolithography, Materials Science Forum 587-588 (2008) 563-567.

[31] P.J. Bartolo, J. Gaspar, Metal filled resin for stereolithography metal part, CIRP Annals Manufacturing Technology, 57 (2008) 235-238.

[32] M. D. Timmer, C. G. Ambrose, A. G. Mikos, In vitro degradation of polymeric networks of poly(propylene fumarate) and the crosslinking macromer poly(propylene fumarate)-diacrylate, Biomaterials 24 (4) (2003) 571 - 577. doi:http://dx.doi.org/10.1016/S0142-9612(02)00368-X. URL http://www.sciencedirect.com/science/article/pii/S014296120200368X

[33] P. Karrer, S. Corbel, J.C. Andre, D.J.Lougnot, Shrinkage effects in photopolymerizable resins containing filling agents: Application to stereophotolithography, Journal of Polymer Science Part A: Polymer Chemistry 30 (13) (1992) 2715-2723. doi:10.1002/pola.1992.080301307. URL http://dx.doi.org/10.1002/pola.1992.080301307

[34] L. A. Hockaday, K. H. Kang, N. W. Colangelo, P. Y. C. Cheung, B. Duan, E. Malone, J. Wu, L. N. Girardi, L. J. Bonassar, H. Lipson, C. C. Chu, J. T. Butcher, Rapid 3d printing of anatomically accurate and mechanically heterogeneous aortic valve hydrogel scaffolds, Biofabrication 4 (3) (2012) 035005. URL http://stacks.iop.org/1758-5090/4/i=3/a=035005

[35] Wang Martha O., Piard Charlotte M., Melchiorri Anthony, Dreher Maureen L., and Fisher John P.. Tissue Engineering Part A. May 2015, 21(9-10): 1642-1653. doi:10.1089/ten.tea.2014.0495. 


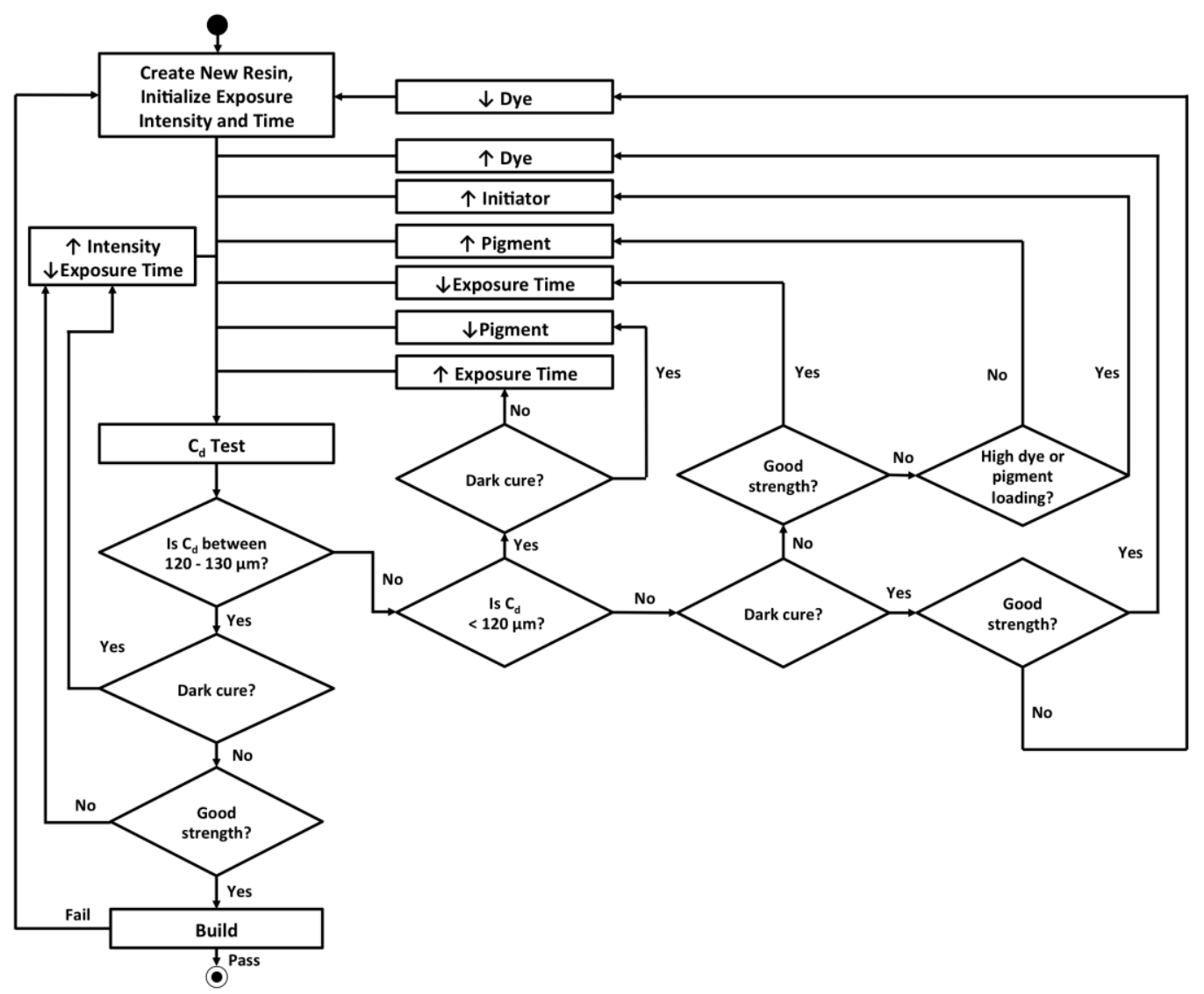

Figure 1: Model of strategy undertaken to identify a working resin given the adjustable additives and build parameters based on the target cure depth, $\mathrm{C}_{\mathrm{d}}$, and $100 \%$ build completion (i.e., the pass condition).
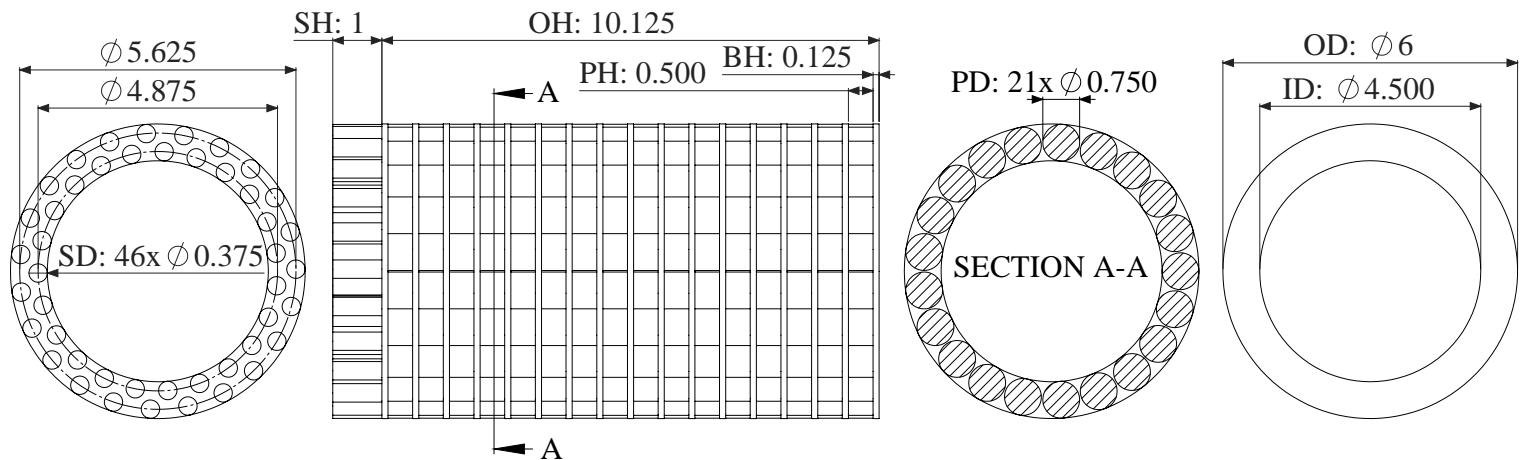

Figure 2: Dimensions of the scaffold design used for benchmarking showing the key features: inner diameter (ID), outer diameter (OD), post diameter $(\mathrm{PD})$, post height $(\mathrm{PH})$, base height $(\mathrm{BH})$, overall height $(\mathrm{OH})$, support diameter $(\mathrm{SD})$, and support height $(\mathrm{SH})$. The build took place from left to right, or support end first. 


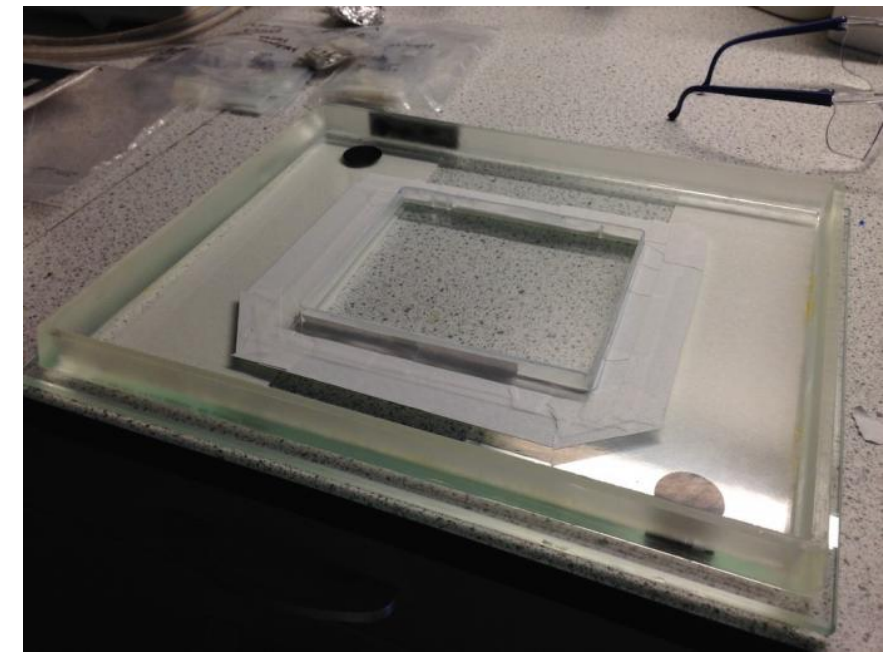

Figure 3: M-type basement modified in order to reduce amount of resin required. 
1

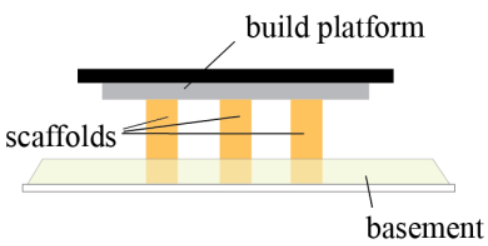

4

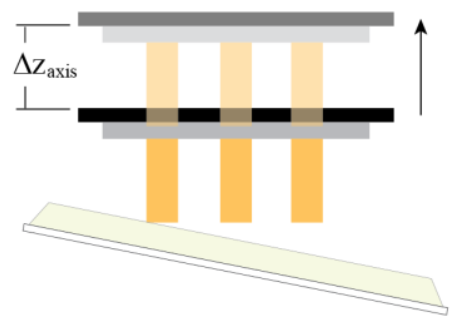

7

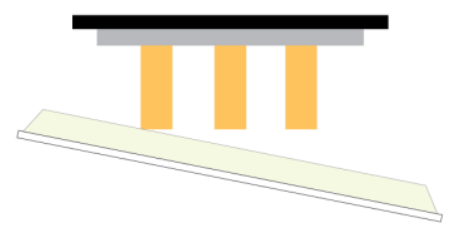

2

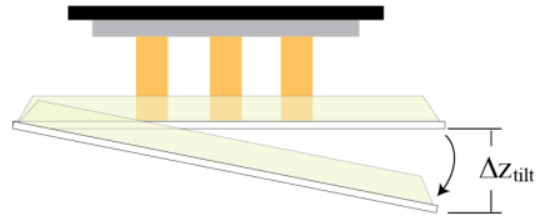

5

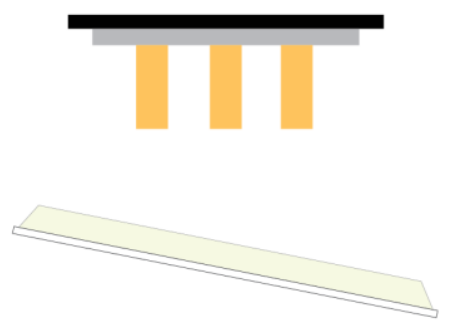

8

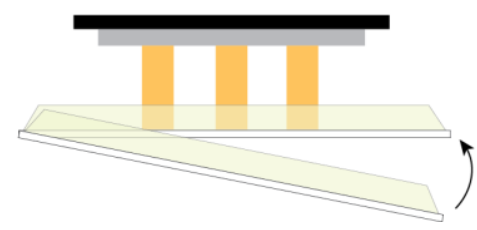

3

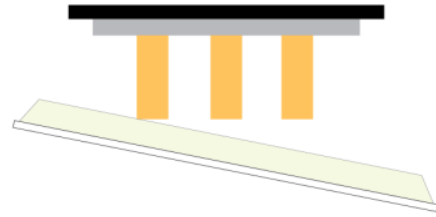

6

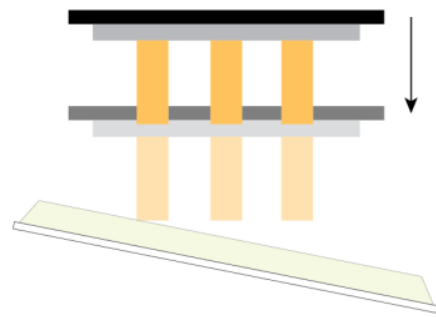

9

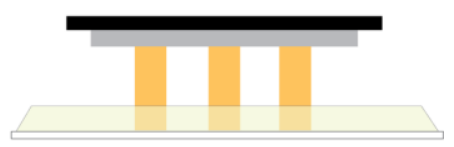

Figure 4: Depiction of the various user-defined build-style parameters in Table 1 (note that movements shown are exaggerated for effect). (1) A waiting time occurs before tilt separation following the completion of a layer of printing. (2) The basement hinges down at the tilt separation velocity to the tilt separation distance, $\Delta \mathrm{z}_{\text {tilt. }}$. (3) Waiting time before axis separation. (4) The build platform moves up at the axis separation velocity to the axis separation distance, $\Delta z_{\text {axis. }}$ (5) Waiting time before axis positioning. (6) The build platform moves down at the axis positioning velocity to position the scaffolds just above the surface of the basement. (7) Waiting time before tilt positioning. (8). The basement hinges up at the tilt positioning velocity to its original level position. The distance between the bottom of the previous built layer of the scaffolds and the surface of the basement is the layer thickness. (9) Waiting time before exposure of the next layer. 


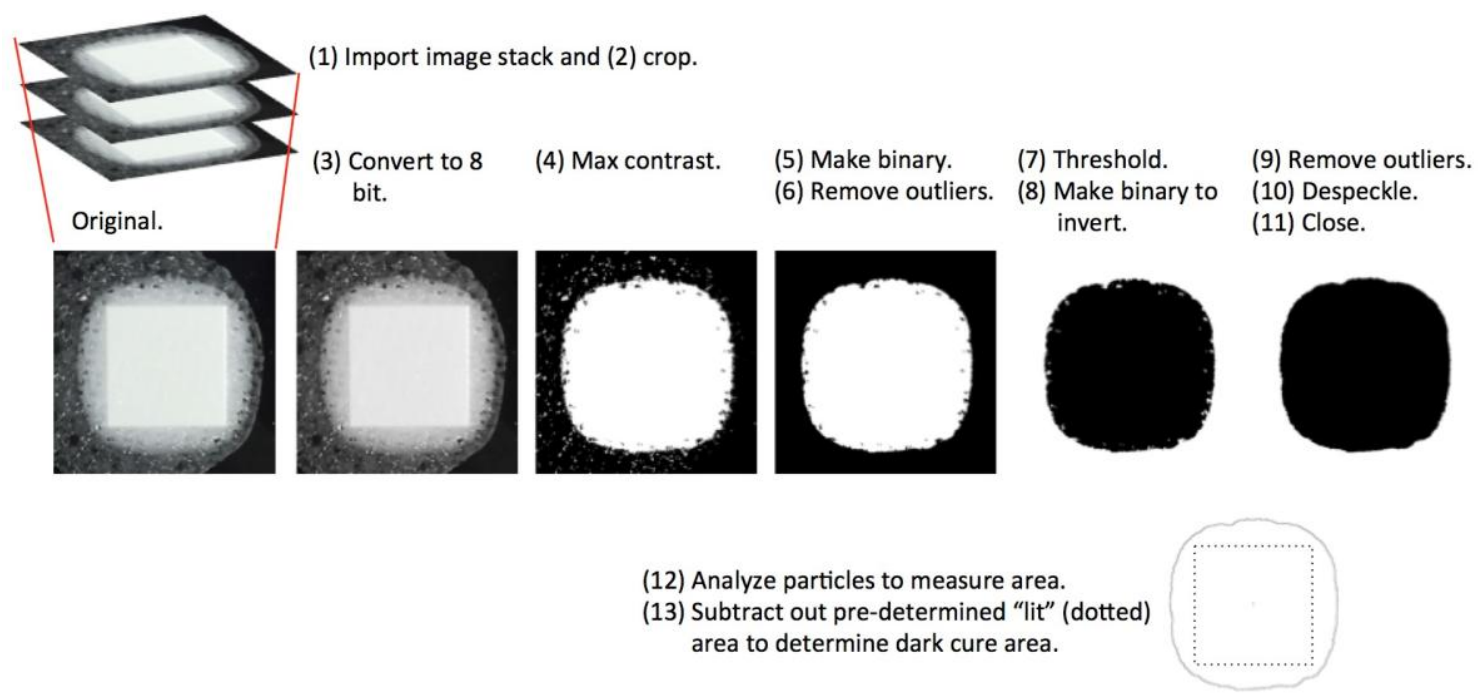

Figure 5: Procedure carried out in ImageJ to analyze the area of dark cure. 


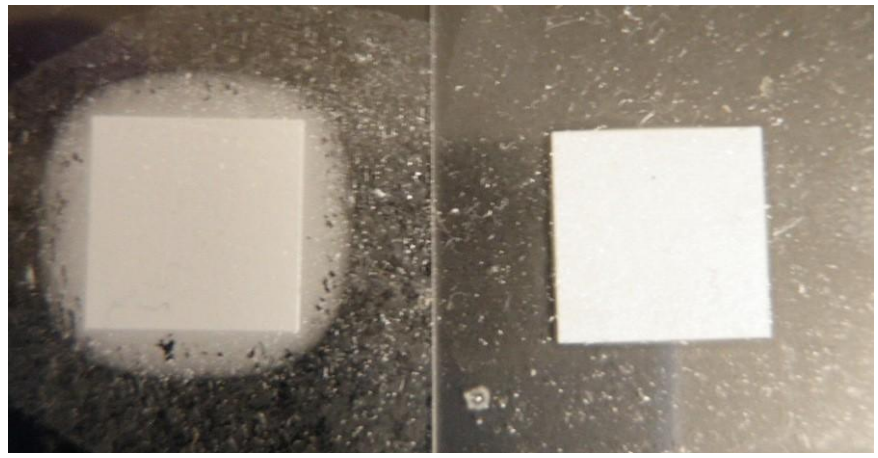

Figure 6: A comparison of two CDTs without (left) and with (right) HMB demonstrating the power of HMB to eliminate dark cure.

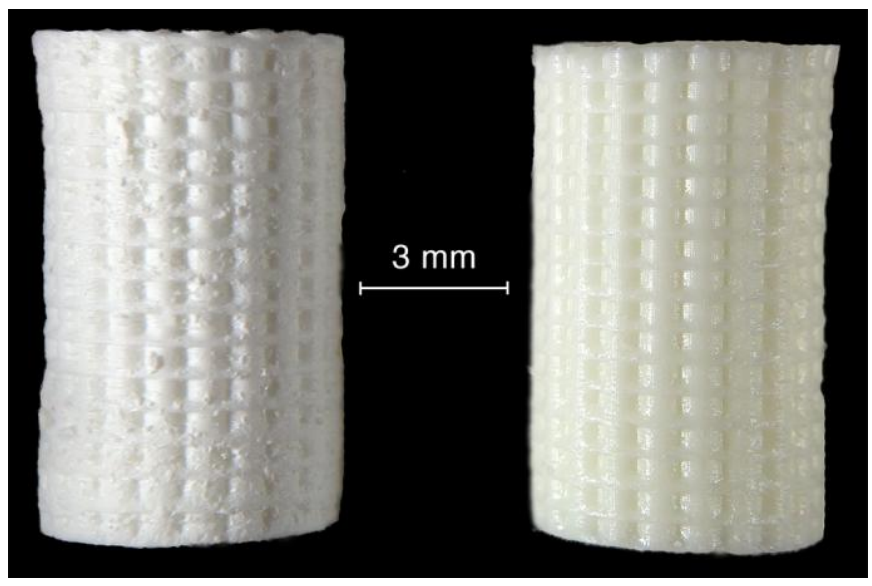

Figure 7: Side-by-side comparison of the same hollow scaffold (Figure 2) built with BR (left) and WR (right). The elimination of particulate buildup through the use of WR is an example of how part accuracy has been increased. These particles have been eliminated through the use of HMB. 


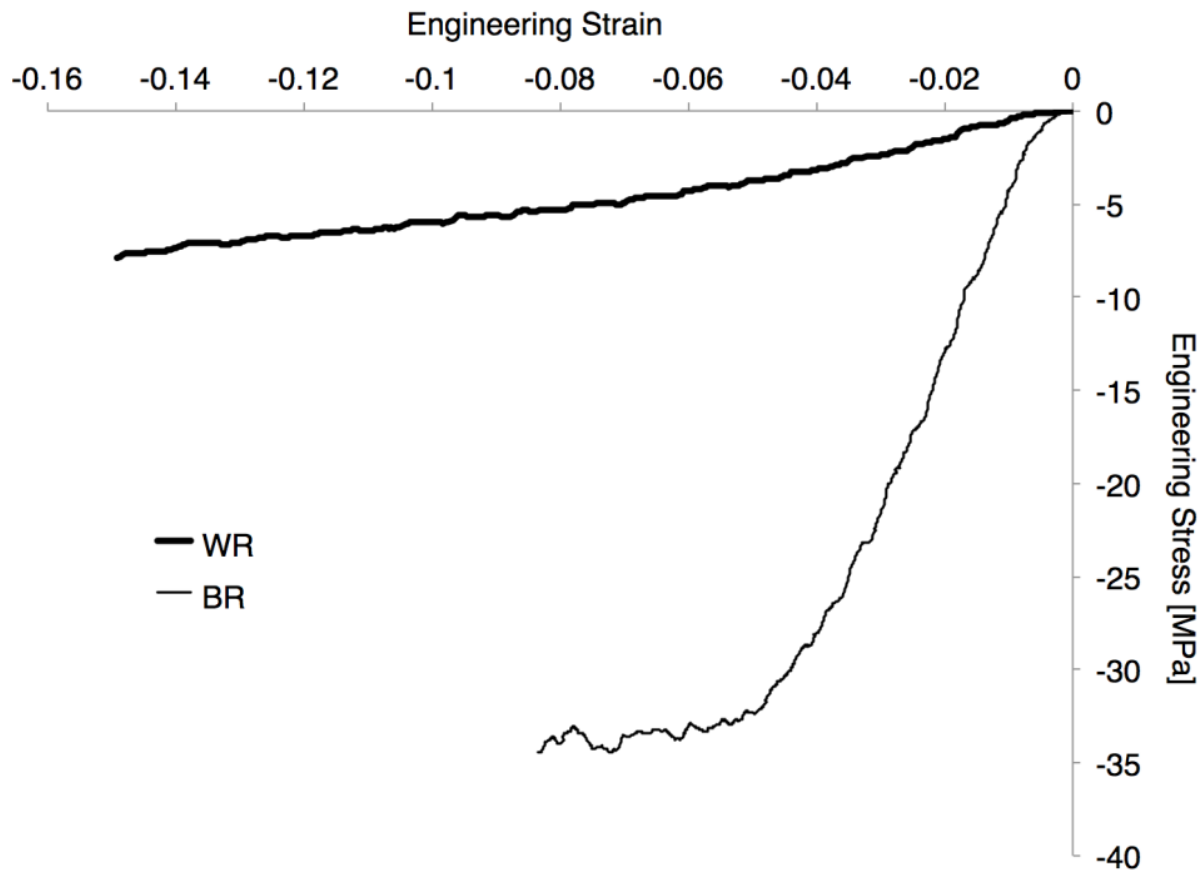

Figure 8: The average stress-strain response of scaffolds built with $B R(n=5)$ and $W R(n=4)$. The failure mode was buckling. It clearly shows the effects of high levels of HMB in WR, which make the scaffolds far more elastic and able to handle larger strains, but at lower stresses.

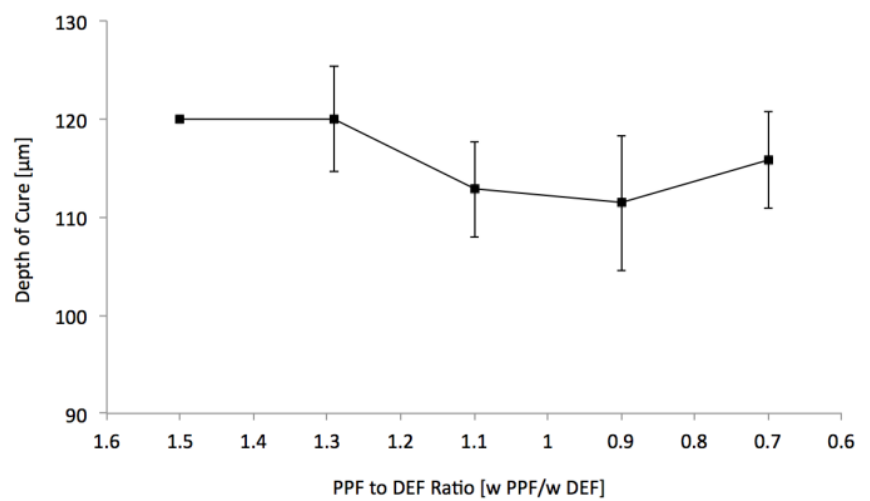

Figure 9: Effect of PPF to DEF ratio on $C_{d}$ for WR. Error bars represent \pm standard error and show that there is no significant change to $C_{d}$ by changing the PPF to DEF ratio $(n=7)$. 


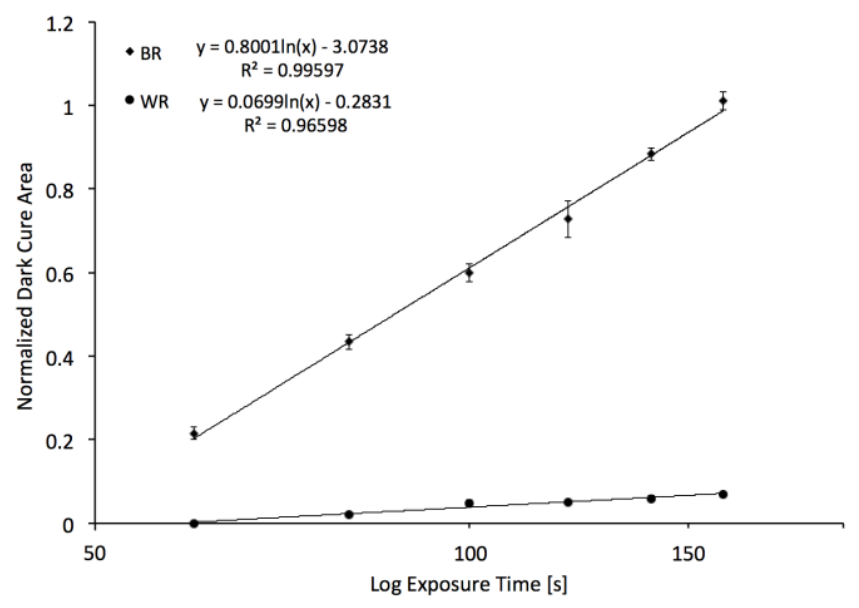

Figure 10: Normalized dark cure area vs. exposure time for WR and BR. Error bars are \pm standard error $(\mathrm{n}=3)$. 

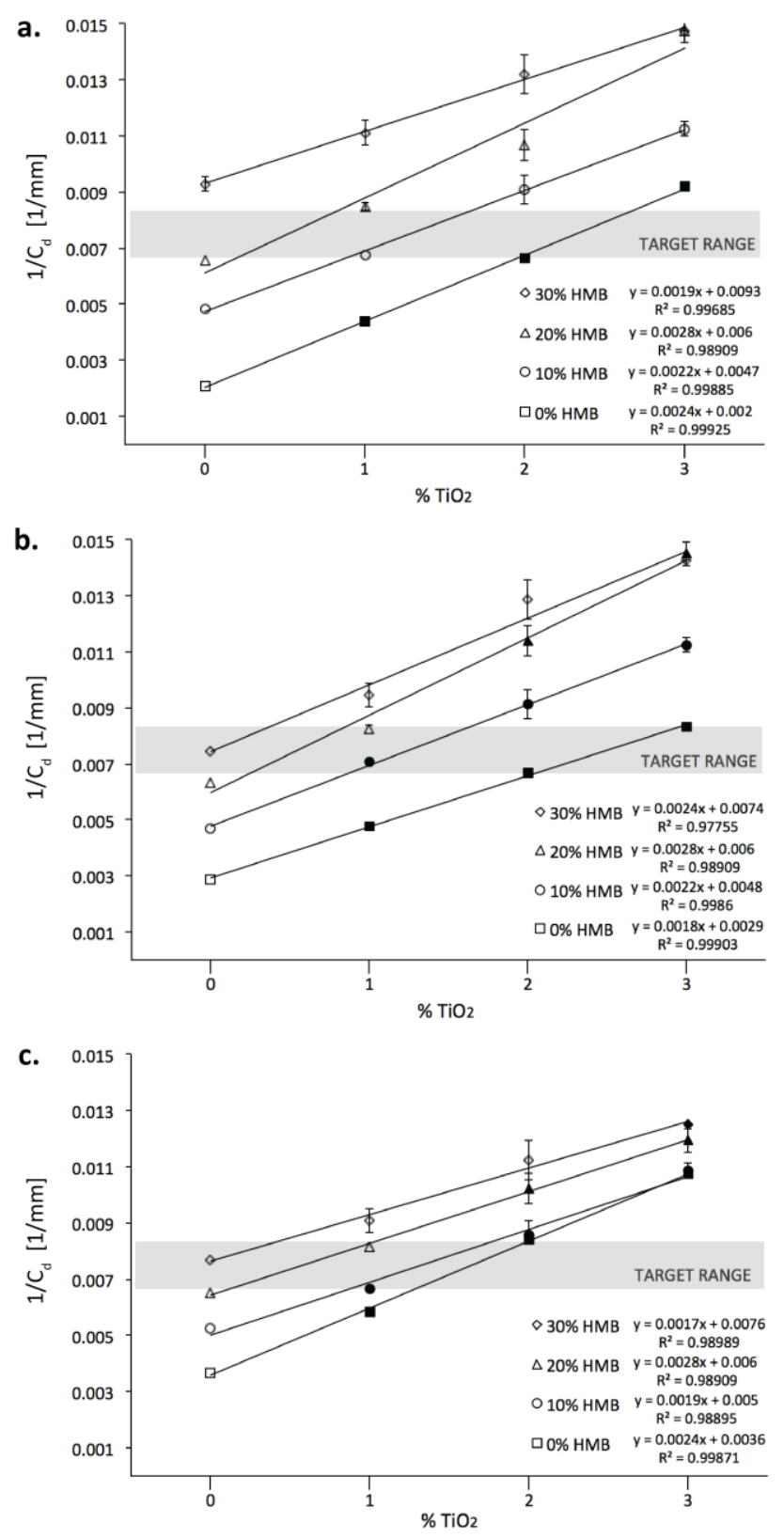

Figure 11: Design space for $\mathrm{C}_{\mathrm{d}}$ using resins with oxybenzone, $\mathrm{TiO}_{2}$, and (a) $1 \% \mathrm{BAPO}$, (b) $2 \% \mathrm{BAPO}$, or (c) $3 \%$ BAPO. The gray zone $(120-150 \mu \mathrm{m})$ is our target range for $\mathrm{C}_{\mathrm{d}}$ to allow for adequate layer-to-layer stitching without compromising resolution. Filled-in markers indicate CDTs that resulted in dark cure. Error bars represent mean \pm standard error $(n=5)$. 

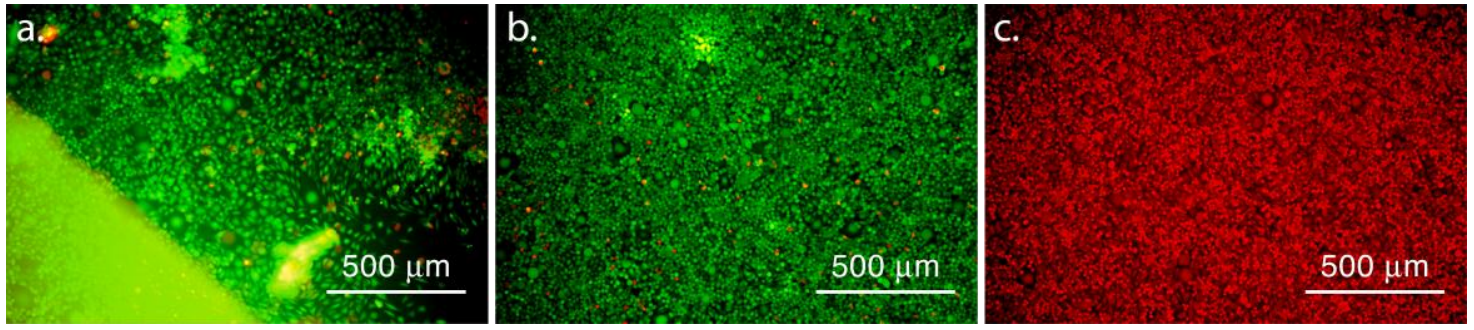

Figure 12: Live/dead staining for cytotoxicity of L929 cells: (a) direct contact with WR thin films, (b) normal culture on polystyrene negative control, and (c) 70\% v/v methanol treated positive control taken at 100x. 
A. Cause of 3D printing problems.

A.

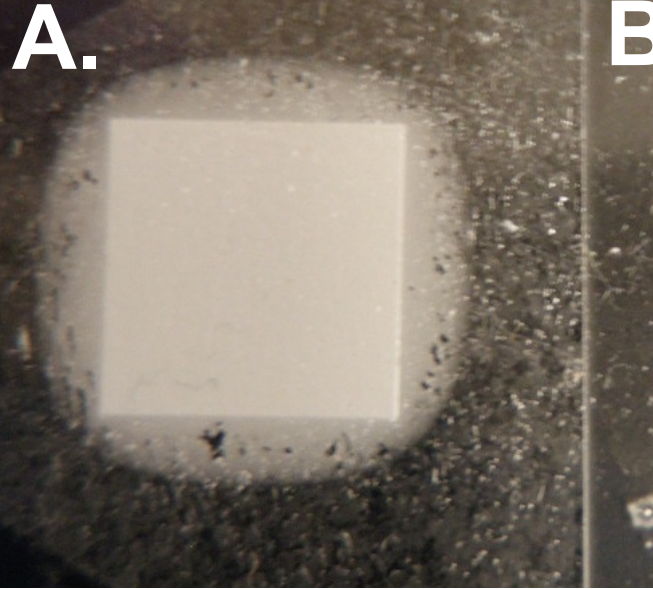

B. New resin with oxybenzone fixed problem.

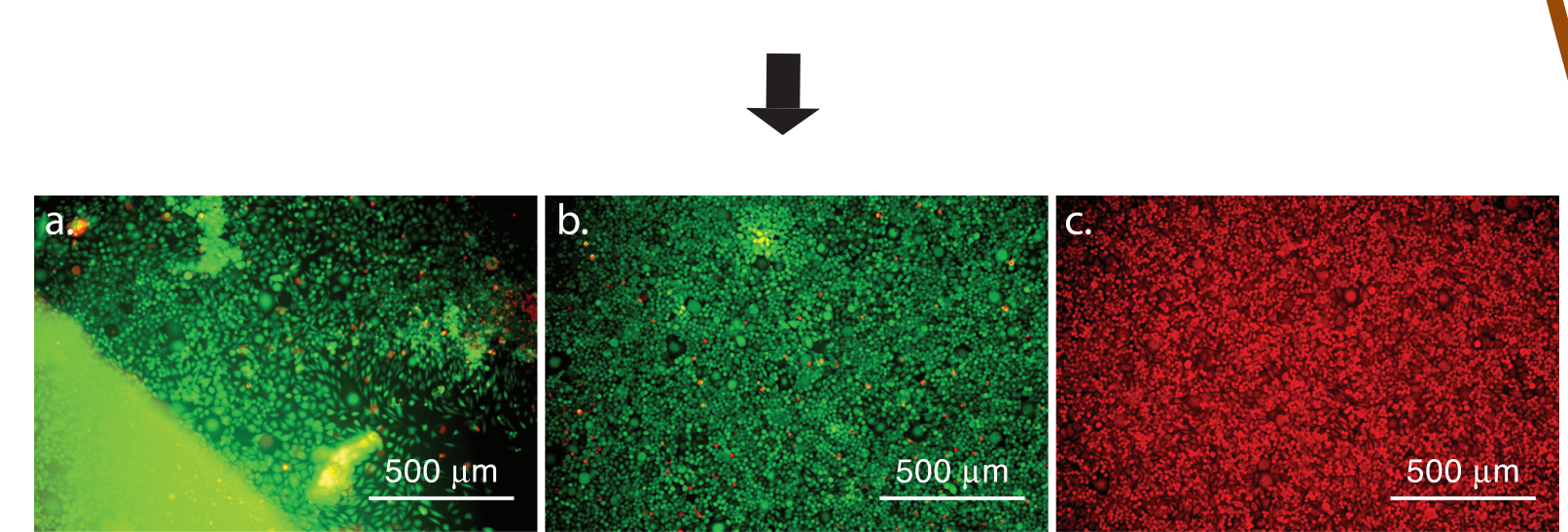

Cytotoxicity test.

B.

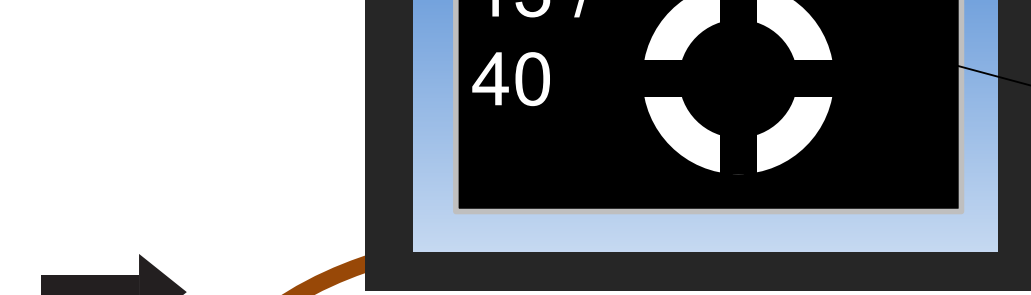

Digital

Photomask

Computer

Build Platform

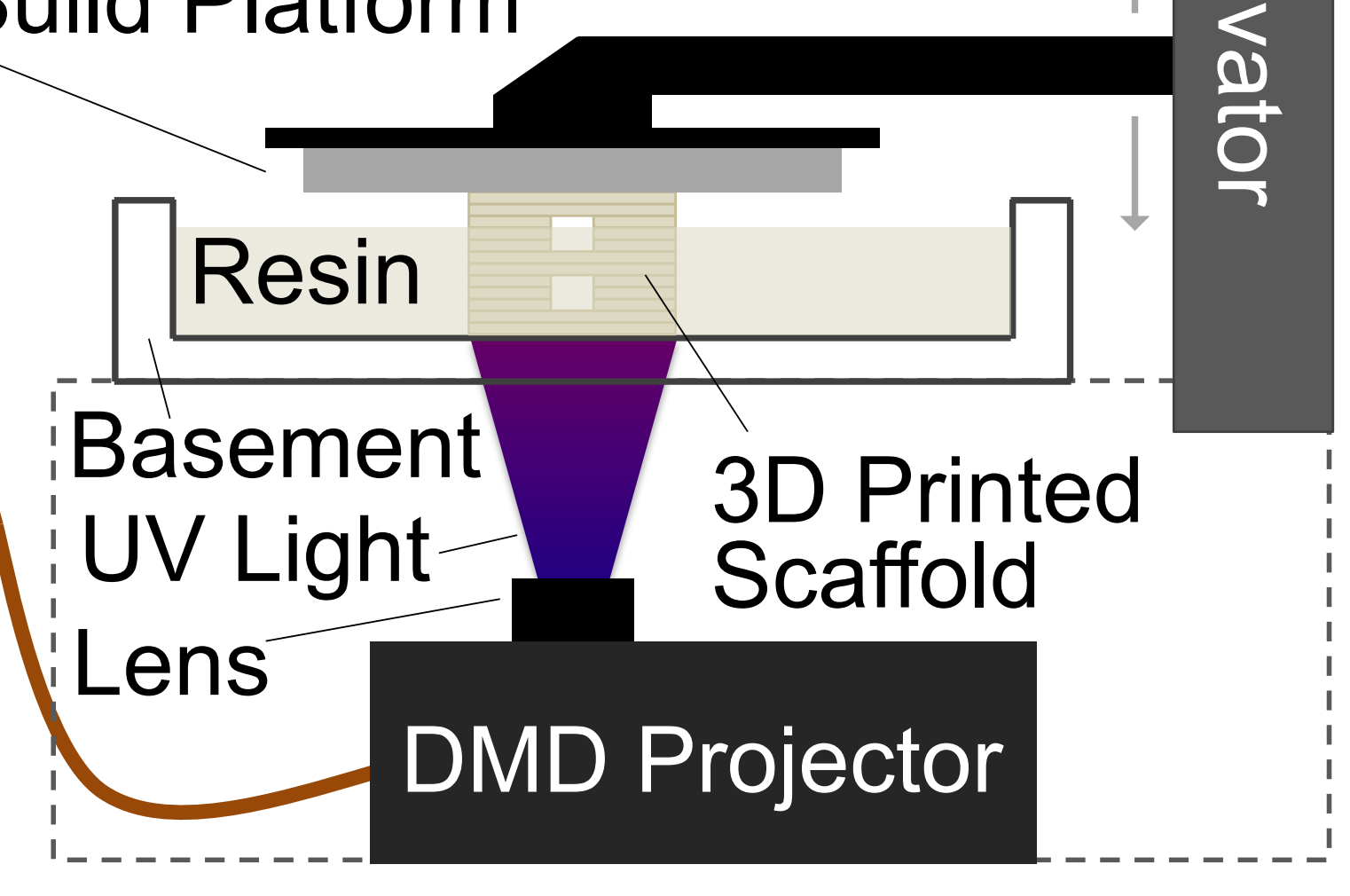

DMD-based 3D printing.

New Resin

Strain

.. New Resin

- Old Resin 